\title{
Body fat as metaphor: from harmful to helpful
}

$\mathrm{T}$ he importance of metaphors in defining clinical reality has long been recognized by social scientists and humanities scholars. In Illness as Metaphor, a highly influential treatise on the topic, Susan Sontag describes how metaphors about tuberculosis, cancer and AIDS shape the way physicians, patients and society see those diseases. ${ }^{1}$ Metaphors influence health: they transform relationships and patient care.

The meanings associated with body fat have changed enormously in recent years, partly because associated metaphors have changed. Body fat was initially seen as an individual trait with moral and/or aesthetic implications. It now falls under medical jurisdiction as an object of practice, research and policy. There are currently two main medical research paradigms dealing with fat bodies, each associated with a different metaphor.

\section{The dominant paradigm: obesity as pathology}

The dominant paradigm sees body fat as pathological. It is seen both as a risk factor for disease and as an actual disease. Pathology as a metaphor for obesity emerged in the late 18th century and has gained traction in recent years. In 1995, the World Health Organization published its technical report 854 , suggesting the worldwide adoption of the body mass index (BMI) categories to define obesity. ${ }^{2}$ The ease with which BMI distributions can be compared across countries and over time led to the publication in 2000 of "Obesity: Preventing and Managing the Global Epidemic." It showed that "obesity" as defined by the new BMI recommendations threatened developed and developing countries alike. Governments worldwide have since tried to monitor and control obesity in their populations and research funding

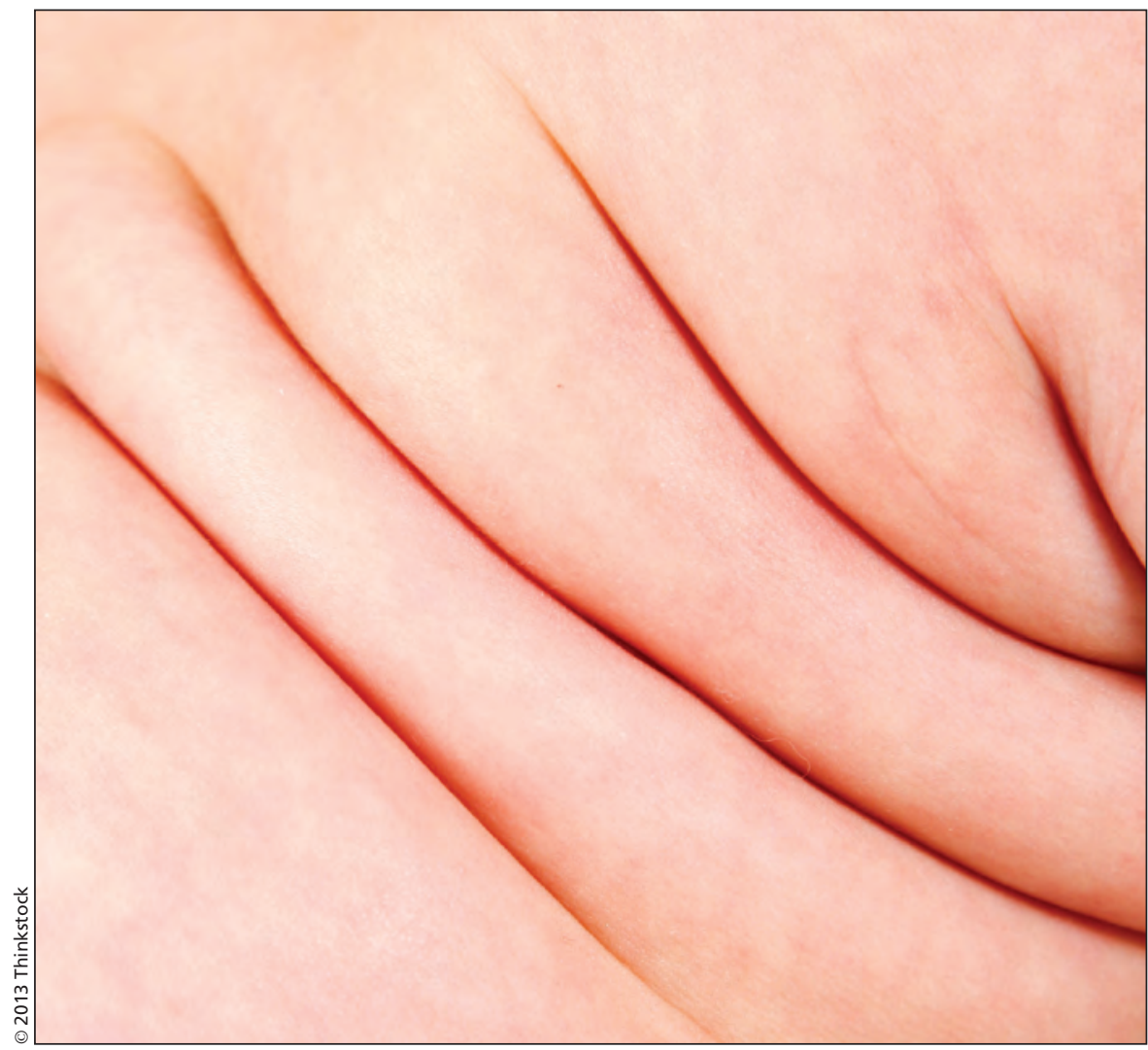

for obesity research has grown along with the number of obesity-related publications.

Researchers working under this paradigm investigate increasing risks of arthritis, certain cancers, cardiovascular disease, diabetes and death for individuals with a BMI above 30 (the definition of obesity). They also actively pursue studies of weight loss, testing the role of drugs, diets, exercise and surgery to "cure" obesity.

\section{The emerging paradigm: obesity as an ascribed characteristic}

This emerging paradigm contests the findings of the dominant paradigm. It highlights its blind spots and suggests that researchers and health practitioners conduct their practices using a "Health at Every Size" (HAES) ${ }^{4}$ approach: one that de-pathologizes body fat and aims to maximize the health of every person, regardless of size; one within which weight loss is never a goal. Researchers and activists within this suggest that we think of fatness as merely another aspect of human diversity, akin to height, race, gender and sexual orientation, rather than as a disease, and argue that the rights of individuals of all sizes should be protected, just as those of women and racial minorities are protected.

This paradigm stresses how our society's fat phobia - its irrational fear of, and disgust with, fat - has led to systematic bias in the majority of current medical research on obesity and to the stigmatization of people who are fat, with serious deleterious health consequences via increased stress, poverty and prejudicial care. ${ }^{5}$ 


\section{Integrating the paradigms}

We do not have to reject all the evidence that correlates obesity and disease to understand the limitations and harmful effects of the dominant paradigm. Fatness, framed as a disease, stigmatizes people who are fat. Framed as an epidemic, it creates cause for alarm and requires action. Beyond alienating patients, these metaphors encourage doctors to evaluate every symptom through a weight-focused lens, and may lead them to ignore important signs of disease and see weight loss as a panacea, ultimately contributing to ill health and early death. A patient who is fat presenting with shortness of breath may, for example, easily be dismissed as too fat for proper breathing function and prescribed weight loss without an examination for other, medically treatable causes of their symptoms.

There is often pushback against nonweight-centred approaches for the health of patients who are fat: Isn't their weight killing them? Recent research suggests that physicians should exercise caution in applying BMI criteria to evaluate the health status of their patients. A 2008 study of BMI and cardiometabolic risk clustering showed that $31.7 \%$ of obese adults in the United States were metabolically "healthy," while as many as $23.5 \%$ of normal-weight adults were "unhealthy." Similarly, a 2011 study showed that BMI barely distinguishes the health risks associated with a BMI of $25 \mathrm{~kg} / \mathrm{m}^{2}$ from those associated with a BMI of $40 \mathrm{~kg} / \mathrm{m}^{2}{ }^{7}$ For someone who is $1.65 \mathrm{~m} \mathrm{(5'6")} \mathrm{tall,} \mathrm{these} \mathrm{BMIs} \mathrm{are}$ equivalent respectively to $68 \mathrm{~kg}$ (150 lbs) and $109 \mathrm{~kg}$ (240 lbs); for someone who is $1.78 \mathrm{~m}$ (5'10”), $79 \mathrm{~kg}$ (174 lbs) and $127 \mathrm{~kg}$ (279 lbs).

Physicians are trusted to provide the best possible care for their patients including encouragement to exercise and eat right - regardless of their race, gender and socioeconomic status. They are also expected to advocate for patients and their health. Why not extend these worthy aims to size? If we were to pay attention to the metaphors we preconsciously call upon when we see patients who are fat, we would be able to acknowledge the biased lens through which we tend to see their health problems and improve our quality of care. Given physicians' power in society, challenging misguided beliefs about body fat could spearhead a cultural transformation.

\section{Elise Paradis MA PhD}

Currie Postdoctoral Fellow

Wilson Centre for Research in Education

University of Toronto

Toronto, Ont.
Ayelet Kuper MD DPhil

Assistant professor

Department of Medicine

Sunnybrook Health Sciences Centre and

Wilson Centre for Research in Education

University of Toronto

Toronto, Ont.

Richard K. Reznick MD MEd

Dean

Faculty of Health Sciences

Queen's University

Kingston, Ont.

\section{References}

1. Sontag S. Illness as metaphor, and AIDS and its metaphors. New York (NY): Picador; 1990.

2. World Health Organization Expert Committee. Physical status: the use and interpretation of anthropometry: report of a WHO Expert Committee. Geneva: World Health Organization; 1995.

3. Obesity: preventing and managing the global epidemic. Report of a WHO consultation. World Health Organization Technical Report Series. 2000; 894:i-xii, 1-253.

4. Bacon L. Health at every size: the surprising truth about your weight. Dallas (TX): BenBella Books; 2010.

5. Puhl RM, Heuer CA. The stigma of obesity: a review and update. Obesity 2009;19:941-64.

6. Wildman RP, Muntner P, Reynolds K, et al. The obese without cardiometabolic risk factor clustering and the normal weight with cardiometabolic risk factor clustering: prevalence and correlates of 2 phenotypes among the US population (NHANES 1999-2004). Arch Intern Med 2008; 168:1617.

7. Padwal RS, Pajewski NM, Allison DB, et al. Using the Edmonton obesity staging system to predict mortality in a population-representative cohort of people with overweight and obesity. CMAJ 2011; 183:E1059-66.

CMAJ 2013. DOI:10.1503/cmaj.120100

\section{More Humanities online}

\section{Poetry}

Steeplechase charity gala, by Ryan E. Childers, Baltimore, Md.; Words of disclosure, by Joshua Liao, Houston, Tx.; Vanishing point, by Wynne Morrison, Philadelphia, Pa.

\section{Books}

The Pain Detective: Every Ache Tells a Story, by Hillel M. Finestone (Praeger Publishers; 2009). This fascinating look at the intricate interplay of mind and body, underscores the importance of emotional and social underpinnings of chronic pain. - Marcin Chwistek, Philadelphia, Pa.

The Country Doctor Revisited: A Twenty-First Century Reader, Therese Zink, editor (Kent State University Press; 2011). Most books about rural physicians focus on the romance of the bygone era; this book takes a broad view of the new rural reality through vignettes, poetry, essays and a blog. - Ian Cameron, Sherbrooke, NS.

\section{Art}

Healing and hurting, all in one building in Mexico. The Museum of Mexican Medicine shares space with the Museum of the Inquisition in Mexico City, meaning that screams of terror accompany visitors through exhibits examining domestic medical practices pre-Hispanic days to the early 20th century. - Paul Gessell, Ottawa, Ont.

Comic relief. Graphic narratives, an intellectual cousin of the comic, offer an accessible medium for patients and providers to express profound experiences - and learn. - Desmond Cole, Toronto, Ont.

CMAJ 2013. DOI:10.1503/cmaj.122102 